\title{
Aerosolised fluorescein can quantify FFP mask faceseal leakage: a cost-effective adaptation to the existing point of care fit-test.
}

\section{Sameer Zaman}

Imperial College London

Henry Seligman

Imperial College London

Freya Hepworth Lloyd

Imperial College London

Keval Tushar Patel

Guy's and St. Thomas' NHS Foundation Trust

\section{Digby Chappell}

Imperial College London

\section{Danny O'Hare}

Imperial College London

\section{Graham D Cole}

Imperial College Healthcare NHS Trust

\section{Darrel P Francis}

Imperial College London

Ricardo Petraco

Imperial College London

Nick W F Linton ( $\nabla$ nick.linton@imperial.ac.uk)

Imperial College London

\section{Research Article}

Keywords: PPE, FFP, fluorescein, filtering masks

Posted Date: November 10th, 2020

DOl: https://doi.org/10.21203/rs.3.rs-104710/v1

License: (a) (i) This work is licensed under a Creative Commons Attribution 4.0 International License.

Read Full License 


\title{
Aerosolised fluorescein can quantify FFP mask faceseal leakage: a cost-effective adaptation to the existing point of care fit-test.
}

\author{
Sameer Zaman', Henry Seligman', Freya Hepworth Lloyd', Keval T Patel'2, Digby Chappell', \\ Danny O'Hare', Graham D Cole ${ }^{3}$, Darrel P Francis ${ }^{1,3}$, Ricardo Petraco ${ }^{1,3}$, Nick W F Linton ${ }^{1,3}$ \\ I. Imperial College, London, UK \\ 2. Guy's and St. Thomas' NHS Foundation Trust, London, UK \\ 3. Imperial College Healthcare NHS Trust, London, UK \\ Corresponding author: \\ Dr. Nick Linton, Department of Bioengineering, Imperial College London, Exhibition Road, London, SW7 2AZ \\ nick.linton@imperial.ac.uk
}

\begin{abstract}
Background: Filtering facepiece (FFP) respirators must provide an adequate faceseal to protect healthcare workers from harmful particles. A qualitative fit-test using bitter-tasting aerosols the commonest way to determine if an FFP mask is safe enough for clinical use. This taste-test is subjective and can be biased by placebo. We propose a cheap and quantitative modification of the taste-test, by measuring the amount of fluorescein staining filter paper behind the FFP mask after a fit-test protocol, using digital image analysis.
\end{abstract}

Methods: Medical grade fluorescein was added to bitter-tasting denatonium benzoate solution and Aerosolised during a mask fit-testing protocol. Scientific filter paper was placed on the inner surface of the mask. Participants were asked if they could taste the solution to determine their qualitative 'pass' or 'fail' result. Filter paper photographs were analysed after the test to quantify total fluorescence (TF). TF levels in the taste-test 'pass' and 'fail' groups were compared.

Results: Fifty-six healthcare professionals completed the fluorescein mask fit-test protocol. 32 (57\%) 'passed' the qualitative (taste) test and the remainder 'failed'. There was a significant difference in TF between the groups based on their qualitative results $(p<0.00 \mathrm{I})$. A cut-off of TF $=5.0 \times 10^{6}$ fluorescence units was determined by analysing the precision (78\%) and recall (84\%) of the fluorescein test. Applying this cut-off resulted in 5 out of 56 participants ( $9 \%$ being reclassified from 'pass' to 'fail' by the fluorescein test. 7 out of 56 (I2\%) participants were reclassified from 'fail' to 'pass'.

Conclusions: Fluorescein is detectable and sensitive to identify faceseal leaks in FFP masks. The fluorescein fit-test is discriminating in its ability to divide people into 'pass' and 'fail' groups similarly to the taste-test. The adaptations are low-cost and could be incorporated in the point-of-care setting. After further validation the fluorescein test could increase safety for staff by reducing the number of false 'pass' by the taste-test. It could also reassure people who have 'failed' the taste-test that they have low levels of fluorescein leak, enabling them to return to clinical practice safely.

Keywords: PPE, FFP, fluorescein, filtering masks 


\section{Background}

Filtering facepiece (FFP) masks are a key item of personal protective equipment (PPE) to reduce transmission of infectious pathogens via aerosols and small droplets (I-3). In light of the SARS CoV-2 global coronavirus pandemic (COVID-19), extensive guidelines have been published recommending the use of FFP masks by healthcare workers (4-7). Many public health bodies mandate healthcare workers to wear an FFP mask - that they have been fittested to use - when undertaking aerosol generating procedures (AGP) or working in areas where these are occurring public $(5,8-10)$.

FFP3 (equivalent to N99 in the USA) masks offer the highest grade of filtration and filter at least $99 \%$ of airborne particles, with an inward leak of approximately $2 \%(\mathrm{II}, \mathrm{I} 2)$. For healthcare professionals to benefit fully from this degree of filtration, any leaks in the mask's seal around the wearer's face must be avoided. Leaks could risk transmission of virus particles carried in aerosols and droplets by bypassing the mask's intrinsic filtration system. Before a mask can be deemed to be safe for use in clinical practice by an individual, a fit-test is undertaken $(8,9)$. Two types of fit-test are available $(13)$ :

(i) Qualitative fit-test (taste-test): A strong-tasting bitter or sweet solution is Aerosolised using handheld pumps near the face of the user whilst they are wearing the mask. The user undertakes a protocolised set of movements. If they perceive the taste, the test is terminated and said mask is deemed unsuitable for their use ('fail'). If they complete the protocol without perceiving an abnormal taste, the mask is deemed to be safe for their use ('pass').

(ii) Quantitative fit-test: Specialist equipment is used by trained practitioners to determine the effectiveness of the faceseal using particle counting.

The taste-test is cheap (under 250 USD)(I4), does not require much training to conduct and is used in almost all centres in the UK. However, it is a subjective test which relies on participants to accurately identify and report an abnormal taste. It can be challenging for participants to be sure that their perceptions are not spurious. Taste can be significantly altered by confounders such as recent food and drink ingestion and environmental smells. 
Furthermore, natural variations in individual sensitivities to smell and taste can contribute to false positives and false negatives. Data from our centre (under peer review) suggest that a significant placebo effect exists, with $23 \%$ of participants reporting 'bitter' or 'sweet' taste during fit-tests when only Aerosolised water was used (I5).

Quantitative fit-tests offer a measurable alternative to the qualitative tests. Obviating the reliance on taste reporting, they use particle counting to determine the effectiveness of the faceseal (13). They must be conducted by trained testers, and are much more expensive than the taste-test, costing in excess of 5000 USD (excluding training)(16). They are believed to be more accurate and have a low 'pass' rate compared to the taste-test $(17,18)$. The quantitative test makes a hole in the mask to enable particle counting on both sides. The item must be discarded after the test, which greatly reduces this test's applicability in resource-strained situations as was seen during the COVID-19 pandemic $(4,19,20)$.

Medical fluorescein is used extensively in clinical practice, most commonly in ophthalmic angiography $(21,22)$. It has been shown to be safe for human inhalation in diagnostic tests (23). Fluorescent sprays and aerosols have previously been used to demonstrate which areas of face masks are likely to contain maximum virus particles (24) and determine the site of faceseal leaks $(25,26)$. Measuring fluorescent staining of filter paper has been used to evaluate aerosol contamination distance during dental procedures (27). In this study we propose a novel fit-test method by applying simple and well-established image analysis techniques to detect the amount of fluorescent staining on filter paper behind FFP masks after a testing protocol using Aerosolised fluorescein. The main objectives of this study are to determine (i) whether fluorescein is detectable in this context and (ii) whether this method is sufficiently discriminating to be used for fit-testing of healthcare workers.

\section{Methods}

Healthcare professionals treating COVID-19 patients were invited to participate in an FFP mask fit-test (28). An industry-standard qualitative mask fit-test protocol (taste-test) commonly used in the UK NHS (29) was modified by the addition of $2 \mathrm{ml}$ of $20 \%$ fluorescein added to $3 \mathrm{ml}$ of $0.2 \%$ denatonium benzoate (F-JAS Ltd., Flint, UK) in a bespoke fit-testing 
solution. The resulting concentration of fluorescein was lower than the concentration that has previously been shown to be safe for inhalation by humans (23).

Two FFP3 masks (3M I863, 3M, Bracknell, UK; 3M Medline NON245IOV, Medline Industries Inc., Warrington, UK) were tested. The choice of mask was determined by stock availability in the clinical areas in which the tests were being conducted.

\section{Fluorescein fit-test}

I. Participant positioning - Each participant was seated in a clean clinical area. A testing hood was placed over their head, initially without them wearing the FFP mask.

2. Establish taste sensitivity - a low concentration ( $0.02 \%)$ of denatonium benzoate (to which fluorescein was not added) was sprayed (using handheld atomizers) through a small hole in the hood, near the participant's mouth and nose until they could taste it. This determined the number of testing solution sprays they receive during the test.

3. Neutralise mouth - the participant rinsed out their mouth with drinking water.

4. Prepare filter paper and mask - The participant opened the mask and turned the inside surface to face upwards. A single sheet of $(70 \mathrm{~mm}$ diameter) general purpose grade laboratory filter paper (King Scientific, Huddersfield, UK) was placed inside the FFP mask using tongs by the tester, near the participant's mouth and nose (Figure I, A).

5. Mask donning - the participant put on the mask, without touching or changing the orientation of the filter paper. The filter paper was not visible from the outside. The hood was replaced over the participant's head (Figure I, B).

6. Fluorescein spraying - the solution of fluorescein and $0.2 \%$ denatonium benzoate was sprayed through a hole in the hood, near the participant's face and nose. The number of sprays was determined by their sensitivity. A standard protocol was followed (normal breathing, deep breathing, head turning, head nodding, talking, bending, normal breathing), with each stage lasting one minute (29).

7. Mask doffing - At the end of the test, the hood was removed and the participant removes the mask without touching the filter paper. The filter paper was collected by the tester using sterile tongs and placed in a labelled specimen bag (Figure I, Panel C).

If the participant completed the test without tasting the solution, they were deemed to 'pass' the taste-test. If they detected the taste at any stage, they were deemed to 'fail' the 
taste-test, but the full protocol was completed to standardise fluorescein exposure between participants. All participants were exposed to 7 minutes of Aerosolised fluorescein and denatonium benzoate.

Filter paper photography and fluorescence analysis

I. Filter paper preparation - two sprays with a pure water atomizer were used to rehumidify the filter papers (Figure I, C)

2. Photography environment - To create fluorescein excitation, filter papers were photographed under a LED bulb lamp. The emitted light was white with a $475 \mathrm{~nm}$ short pass filter (Edmund Optics Ltd., York, UK). Light was received using a long pass filter at approximately 500nm (Hoya F3 yellow lens filter, Hoya Vision Care Company, Bangkok, Thailand) (Figure I, D).

3. Camera settings - A Canon EOS 700D camera with $18-55 \mathrm{~mm}$ lens (Canon Inc., Tokyo, Japan) was used for photography. The camera settings were ISO: 1600; shutter speed: 0.25 seconds; aperture: $F=8.0$; white balance: 'halogen'.

4. Fluorescence quantification - The photograph was split into the red/green/blue (RGB) stack and the green channel selected using the Fiji distribution of ImageJ (30). The technique described by Hammond ( $3 \mathrm{I}$ ) was adapted to calculate total fluorescence (TF) (Figure I, D).

\section{Statistical analyses}

Median TF was calculated for the 'pass' and 'fail' groups based on participants' taste-test result (Figure I, E). The difference between median TF between the two groups was evaluated using the Mann Whitney $U$ test due to their distributions being non-parametric. The precision and recall of the fluorescein test in comparison to the taste-test was calculated for a range of possible TF thresholds. Maximising both (with a preference for recall) was used to determine a TF cut-off for the fluorescein test. 


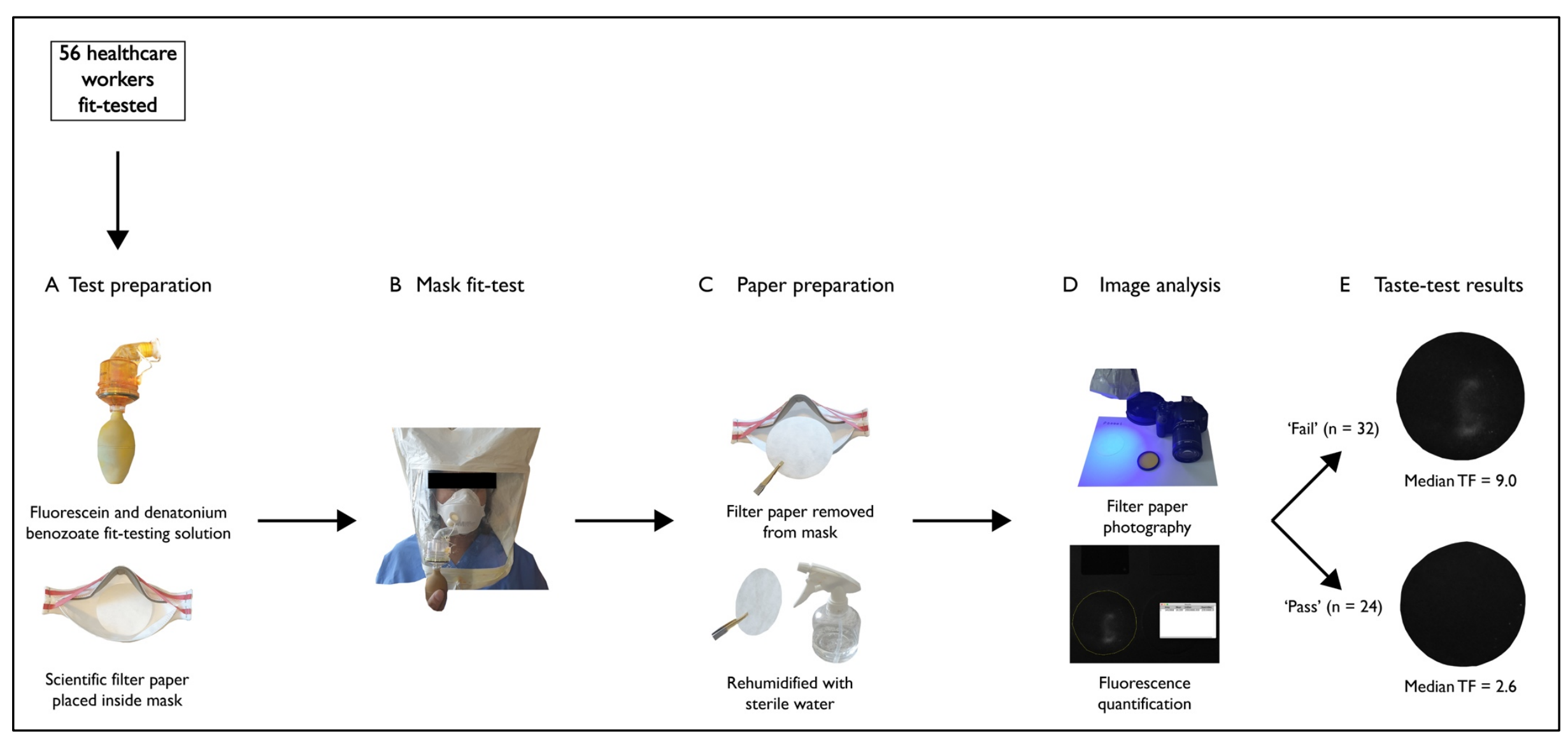

Figure 1 


\section{Results}

Fifty-six healthcare professionals underwent the fluorescein fit-test at our hospital in London, UK. Of these 32 (57\%) 'passed' the taste-test (i.e. they completed the protocol without tasting the solution) and 24 (43\%) 'failed' the taste-test (i.e. they experienced an abnormal perception of taste at some point during the taste protocol). There were comparable baseline characteristics between the taste 'pass' and 'fail' groups (Table I).

In the ungrouped analysis, the median TF of the entire cohort was $3.7 \times 10^{6}$ fluorescence units $\left(I Q R=8.0 \times 10^{6}\right.$ fluorescence units). When participants were stratified by the outcome of their taste-test, there was a significant difference in TF between the 'pass' and 'fail' groups (median TF 'pass' group $2.6 \times 10^{6}(I Q R=2.9)$ fluorescence units vs. median TF in 'fail' group $9.0 \times 10^{6}(I Q R=12.6)$ fluorescence units) $(\mathrm{P}<0.00 \mathrm{I})$. (Figure 2)

Analysis of different TF thresholds on the precision and recall of the fluorescein test in comparison to the taste-test determined a TF of $5.0 \times 10^{6}$ fluorescence units as the optimal cut-off value to separate the 'pass' and 'fail' groups for this dataset (precision $=78 \%$; recall $=$ 84\%) (Figure 3). Applying this value as the result threshold of the fluorescein test resulted in $12(21 \%)$ of participants' taste-test results being overturned (5 out of 56 (9\%) 'pass' changed to 'fail'; 7 out of 56 (I2\%) 'fail' changed to 'pass').

Table 1

\begin{tabular}{|c|c|c|c|}
\hline & All & Taste-test 'pass' & Taste-test 'fail' \\
\hline $\begin{array}{l}\text { Number of participants } \\
\text { Mean age } \\
\text { \% Female }\end{array}$ & $\begin{array}{c}56 \\
36.3 \\
58.9\end{array}$ & $\begin{array}{c}32 \\
37 \\
59.4\end{array}$ & $\begin{array}{c}24 \\
35.5 \\
58.3\end{array}$ \\
\hline $\begin{array}{l}\text { Role } \\
\text { Doctor } \\
\text { Nurse } \\
\text { Allied health professional }\end{array}$ & $\begin{array}{l}21(37.5 \%) \\
23(41.1 \%) \\
12(21.4 \%)\end{array}$ & $\begin{array}{l}\text { I } 6(50.0 \%) \\
12(37.5 \%) \\
4(12.5 \%)\end{array}$ & $\begin{array}{c}9(37.5 \%) \\
10(41.7 \%) \\
5(20.8 \%)\end{array}$ \\
\hline FFP3 mask type & & & \\
\hline $3 M 1863$ & $36(64.3 \%)$ & $21(65.6 \%)$ & 15 (62.5\%) \\
\hline $\begin{array}{l}\text { Medline cone mask } \\
\text { Sensitivity to denatonium benzoate }\end{array}$ & $20(35.7 \%)$ & II (34.4\%) & $9(37.5 \%)$ \\
\hline Normal & $5 I(9 \mid .2 \%)$ & $28(87.5 \%)$ & $23(95.8 \%)$ \\
\hline Low & $4(7.1 \%)$ & $3(9.4 \%)$ & I (4.2\%) \\
\hline Minimal & I (I.7\%) & I (3.1\%) & 0 \\
\hline
\end{tabular}




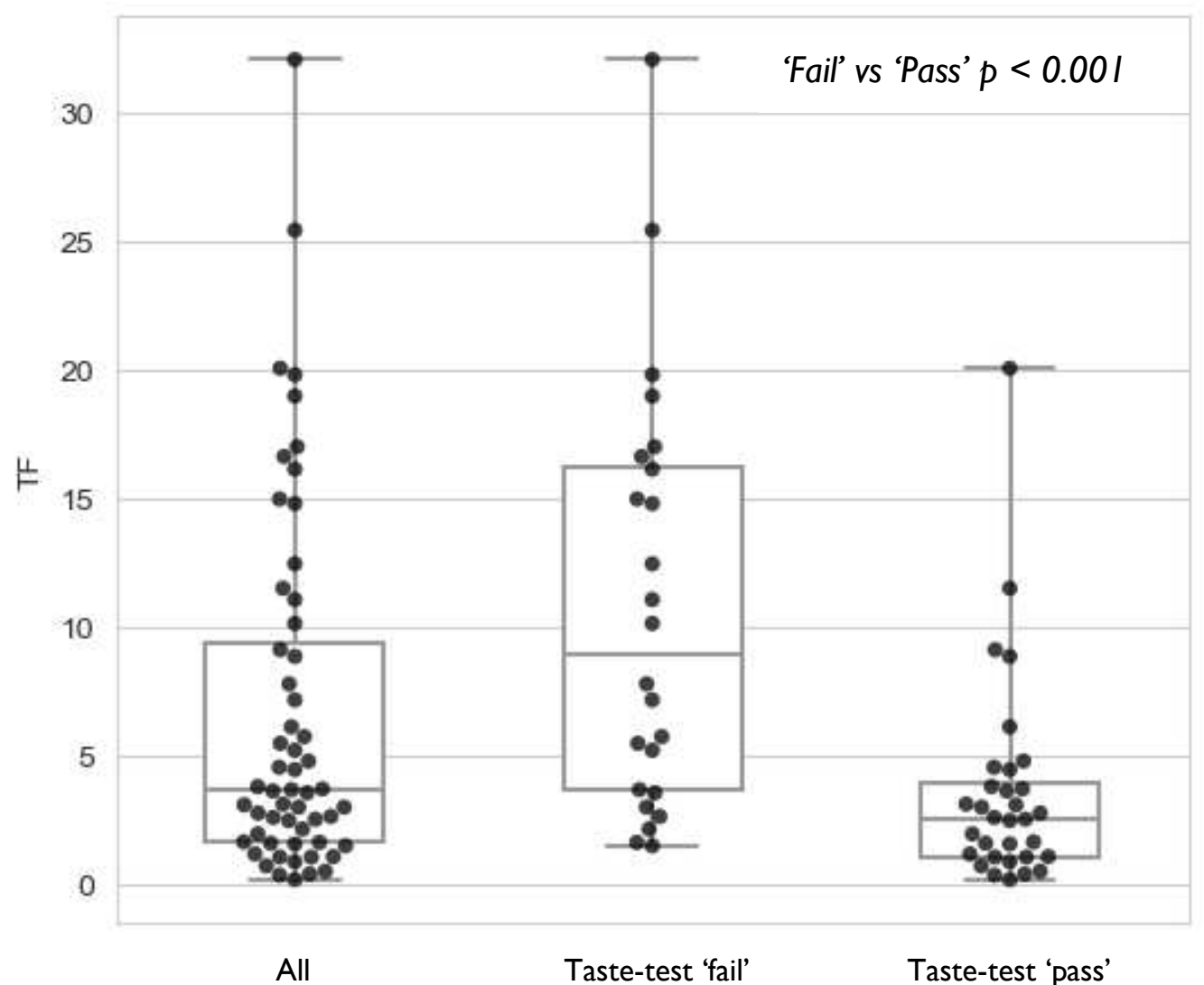

Figure 2

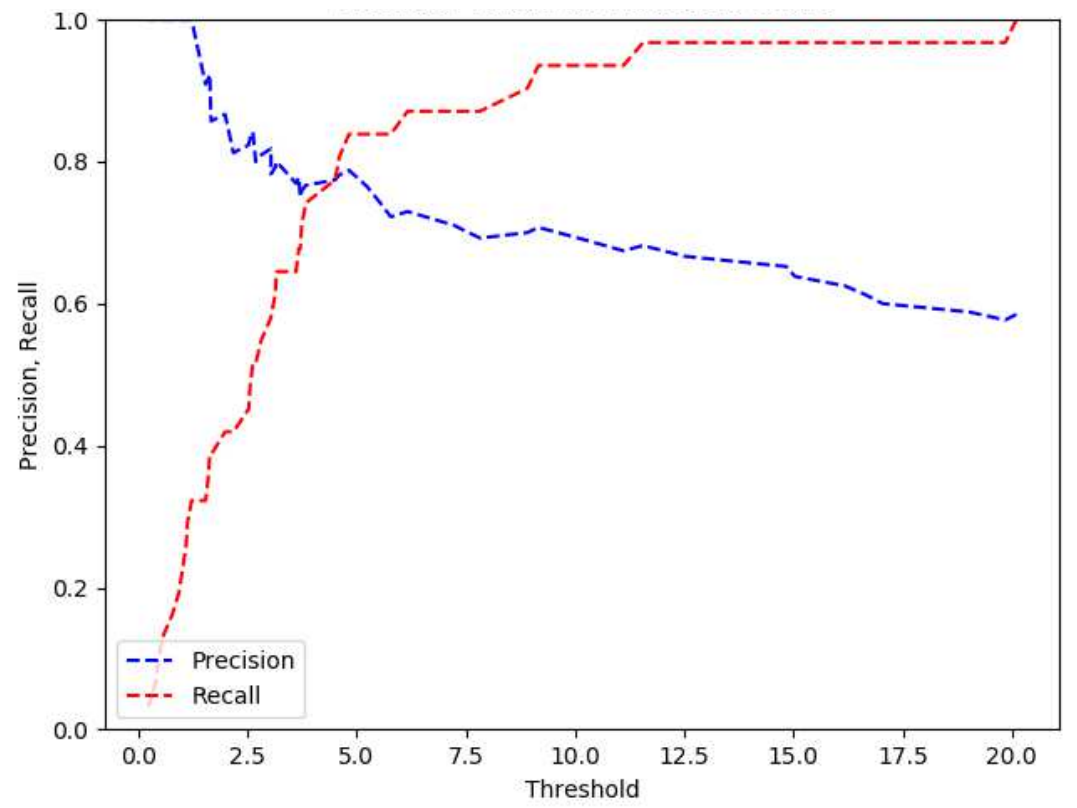

Figure 3 


\section{Discussion}

In this study we introduce a novel, quantitative fluorescein mask fit-test to determine whether FFP respirators adequately fit healthcare workers before their use in clinical practice. This method is an adaptation of the qualitative taste-test using cheap and readily available materials such as medical fluorescein and scientific filter paper. This study achieved its two main objectives by demonstrating that (i) fluorescein is detectable in this context and (ii) the test is sufficiently discriminating to classify users into distinct groups.

Compared to the taste-test, the fluorescein test required no additional time to conduct, and no side effects were experienced by any participants. The fluorescein test is conducted at approximately one tenth of the cost of the alternative quantitative test, and without the need for specialist trained staff or destruction of the mask during testing - a key resourcemanagement benefit in the context of a global viral pandemic.

We have demonstrated that this test can separate users into 'pass' and 'fail' categories similarly to the existing taste-test $(p<0.001)$ : people who could readily taste the bitter aerosols had a high TF and vice versa. Analysing these results from another point of view, they also validate the existing taste-test, which performs reasonably well at classifying users (19/24 participants who were classified as 'pass' by the qualitative test truly did have low TF (less than $5.0 \times 10^{6}$ fluorescence units)).

The main benefit of the fluorescein test is that it provides a numerical output, TF. This mitigates the greatest source of bias in the taste-test: reliance on the user's subjective perception of taste. It cannot be biased by placebo, which has been observed in almost a quarter of the taste-tests in our experience (I5). TF has many positive implications including the comparison of different masks' performance for an individual, quantifying the severity of the leak caused by ill-fitting masks and measuring the effect of finer mask adjustments to overcome leaks. These attributes demonstrate the test's valuable ability to flex with unexpected changes in the PPE supply chain and clinical staff redeployment, as seen in the primary surge of the COVID-19 pandemic $(4,19,20)$.

A key utility of a quantifiable metric of mask-leakage such as TF is the ability to use cut-offs to threshold the test result. This can enable the reclassification of users who may have been 
misclassified by the taste-test. In this study, a the precision and recall of the fluorescein test in comparison to the taste-test was calculated for different TF threshold values. A TF cut-off of $5.0 \times 10^{6}$ fluorescence units is suggested for this dataset because it achieves good precision (78\%) and recall (84\%). Due to the clinical implications of a higher false positive rate, recall was prioritised over precision when considering the cut-off value. Although further validation with a large sample size is required to determine a robust cut-off, adopting this as a threshold for the fluorescein test would result in $9 \%$ of participants who had previously been classed as 'pass' by the taste-test now being classed as 'fail'. This is a group of people who were sure that they did not taste the bitter aerosols during the full 7 minutes of the protocol, yet the filter papers behind their mask were substantially stained with fluorescein. Given that the background environmental fluorescence is negligible, the most likely explanation is that the fluorescein entered through a leak in their masks' faceseals. Ordinarily this group of healthcare workers would have commenced or continued clinical practice wearing that mask, including participation in risky aerosol generating procedures without the benefit of this information, falsely reassured by their results in the taste-test.

Healthcare professionals are disproportionately affected by COVID-19 (32). The implications of reclassifying this group may have considerable impact on their safety and wellbeing by enabling their exclusion from high-risk areas, and the identification of more suitable FFP respirators (33). Unlike the taste-test, the fluorescein test provides this crucial information that has as yet been unavailable to healthcare workers and their managers.

The TF distribution of participants who 'failed' the taste-test (i.e. they reported tasting the bitter aerosols) has a very wide range. On one end of the spectrum, $38 \%$ recorded very high TF levels in excess of $15 \times 10^{6}$ fluorescence units. This group of people not only tasted the solution, but also truly had substantial leaks in the faceseal resulting in filter paper staining. This demonstrates that both the taste-test and fluorescein test were effective in identifying this group. On the other end of the spectrum, $29 \%$ had low levels of TF $(<5.0 x$ $10^{6}$ fluorescence units), despite being certain that they tasted the solution. This can only be explained by either a placebo effect or a heightened sensitivity to tasting denatonium benzoate. Data from our experiments indicate that a placebo effect occurred in $23 \%$ of taste-tests. This group ( $12 \%$ of our entire cohort) could be presented with the results of their fluorescein fit-test and reassured that they actually had low levels of faceseal leak. This 
could enable a substantial proportion of the healthcare workforce to return to patientfacing roles if they were shielded or reduce the need for wearing uncomfortable and expensive respirators. In both cases there would be a positive impact on public health, workforce productivity and human resource management (34).

\section{Limitations}

Although this study can be viewed as proof of concept of a novel and important adaptation to improve the existing FFP mask fit-test, this study has a number of limitations to which future research should be directed. First, the quantitative fit-test was not used as the goldstandard comparator in this study. This was due to its limited availability and high cost during the peak of the COVID-I 9 pandemic. Furthermore, testing masks with this method would have resulted in their subsequent wastage, which was inappropriate in the context of PPE stock shortages during the pandemic. We recommend that the fluorescein test be validated in comparison to the quantitative test to benchmark its performance, and to determine the ideal TF cut-off value.

Second, we tested two commonly used FFP3 masks by two manufacturers. This was based on stock levels and availability in clinical areas when the tests were being conducted. Although the two masks used have different shapes, their coverage is by no means exhaustive. We recommend future research to validate our testing method on a wider range of masks representative of the entire marketplace.

Third, although we have demonstrated that the presence of fluorescein relates to the presence and amount of leak, in this study we have not determined (at an individual level) where the leak is likely to be occurring. In clinical practice, this would be a very helpful piece of information, enabling users to identify the likely site of the mask ill-fitment and the important opportunity to overcome the leak for example by adjustment of tightening straps on the mask or moulding of the wire nasal bridge (35). This could prevent unnecessary escalation to larger and more expensive respirators due to false fit-test failures. We recommend future studies to incorporate a measure of leak location in their protocol, such as marking the filter paper to correspond with facial anatomical landmarks. Commonly identified leak sites could guide future mask development. 
Fourth, in this study the photography and image analysis of the filter papers were done spatially and temporally removed from the fit-testing encounter. This was to identify the ideal photography environment through iterative experimentation. Now that the photographic parameters have been identified in this study, further validation is required to ensure these can recreated with a range of devices to enable point-of-care photography. The image analysis could also be rapidly conducted on a portable computer or smart device. We aim to develop this environment as a point-of-care product and invite further validation.

\section{Conclusions}

This study shows that Aerosolised fluorescein is detectable on filter-paper placed behind FFP masks when added to the existing qualitative fit-test protocol (the taste-test).

Fluorescent staining is sensitive in this context and can be quantified to produce a metric of leakage, TF. The fluorescein test has a similar discriminatory ability to the existing taste-test, and is much cheaper than the quantitative test.

We advocate using fluorescein in mask fit-testing to provide increased safety for 'false passes' in the taste-test ( $9 \%$ in our cohort). After further validation, 'false fails' in the tastetest may be reassured that this is most likely due to a placebo rather than substantial fluorescein leakage ( $12 \%$ in our cohort). This could enable them to safely return to clinical practice.

\section{List of abbreviations (in order of use)}

FFP - filtering facepiece

TF - total fluorescence

PPE - personal protective equipment

AGP - aerosol generating procedure

\section{Declarations}

Ethics approval and consent to participate

This study was approved by Imperial College Healthcare NHS Trust clinical audit and quality improvement committee (Ref CAR_038). Written informed consent was obtained from all participants. 
Consent for publication

Not applicable

Availability of data and materials

The datasets used and/or analysed during the current study are available from the corresponding author on reasonable request

\section{Competing interests}

The authors declare that they have no competing interests.

Funding

Funding for this study was provided by a grant from the Imperial College COVID Research Fund (grant WHCP G26284). We are grateful for the infrastructural support from the National Institute of Health Research (NIHR) Biomedical Research Centre based at Imperial College Healthcare NHS Trust and Imperial College London.

\section{Authors' contributions}

SZ, HS, DO, RP and NWPL conceived the research question and study protocol, performed data analyses and were major contributors in writing the manuscript. FHL and KTP conducted primary data collection. DC, GDC and DPF conducted statistical analyses and contributed in manuscript writing. All authors read and approved the final manuscript.

\section{Acknowledgements}

Not applicable 


\section{References}

I. O'Dowd K, Nair KM, Forouzandeh P, Mathew S, Grant J, Moran R, et al. Face Masks and Respirators in the Fight against the COVID-19 Pandemic: A Review of Current Materials, Advances and Future Perspectives. Materials (Basel). 2020 Jul 29; I3(I5).

2. Sommerstein R, Fux CA, Vuichard-Gysin D, Abbas M, Marschall J, Balmelli C, et al. Risk of SARS-CoV-2 transmission by aerosols, the rational use of masks, and protection of healthcare workers from COVID-19. Antimicrob Resist Infect Control. 2020 06;9(I):100.

3. Bin-Reza F, Lopez Chavarrias V, Nicoll A, Chamberland ME. The use of masks and respirators to prevent transmission of influenza: a systematic review of the scientific evidence. Influenza Other Respir Viruses. 2012 Jul;6(4):257-67.

4. World Health Organization (WHO). Rational use of personal protective equipment for coronavirus disease (COVID-19) and considerations during severe shortages [Internet]. WHO; 2020 [cited 2020 Sep 2]. Available from: https://apps.who.int/iris/bitstream/handle/ I0665/33 I695/WHO-20I9-nCovIPC_PPE_use-2020.3-eng.pdf? sequence=9\&isAllowed=y

5. Centers for Disease Control and Prevention. Interim Infection Prevention and Control Recommendations for Healthcare Personnel During the Coronavirus Disease 2019 (COVID-19) Pandemic [Internet]. CDC; 2020. Available from: https://www.cdc.gov/coronavirus/2019-ncov/hcp/infection-controlrecommendations.html

6. Public Health England. New government recommendations for England NHS hospital trusts and private hospital providers [Internet]. UK Government; 2020 [cited 2020 Sep 2]. Available from: https://www.gov.uk/government/publications/wuhan-novelcoronavirus-infection-prevention-and-control/new-government-recommendations-forengland-nhs-hospital-trusts-and-private-hospital-providers

7. Lepelletier D, Grandbastien B, Romano-Bertrand S, Aho S, Chidiac C, Géhanno J-F, et al. What face mask for what use in the context of COVID-19 pandemic? The French guidelines. J Hosp Infect. 2020 Apr 26;

8. Public Health England. Putting on (donning) personal protective equipment (PPE) for aerosol generating procedures (AGPs). Alrbone Precautions - Gown Version [Internet]. UK Government; 2020. Available from:

https://assets.publishing.service.gov.uk/government/uploads/system/uploads/attachment _data/file/9II333/PHE_COVID-

19_Donning_Airborne_Precautions_gown_version__003_.pdf

9. NHS England. FAQs on using FFP 3 Respiratory Protective Equipment (RPE) [Internet]. NHS England; 2020 [cited 2020 Sep 2]. Available from:

https://www.england.nhs.uk/coronavirus/wp-content/uploads/sites/52/2020/03/faq-ffp324-march-2020.pdf

10. World Health Organization (WHO). Infection prevention and control of epidemic- and pandemic-prone acute respiratory diseases in health care [Internet]. WHO; 2007 
[cited 2020 Sep 2]. Available from:

https://apps.who.int/iris/bitstream/handle/I0665/69707/WHO_CDS_EPR_2007.6_eng.P

df? sequence $=$ I

II. Alexander, John. N95 vs FFP3 \& FFP2 masks - what's the difference? [Internet]. Fast Life Hacks. 2020 [cited 2020 Sep 2]. Available from: https://fastlifehacks.com/n95-vs-ffp/

12. Greenhalgh, Trisha, Chan, Xin Hui, Khunti, Kamlesh, Durand-Moreau, Quentin, Straube, Sebastian, Devane, Declan, et al. What is the efficacy of standard face masks compared to respirator masks in preventing COVIDtype respiratory illnesses in primary care staff? [Internet]. The Centre fo Evidence-Based Medicine; 2020 [cited 2020 Sep 2]. Available from: https://www.cebm.net/covid-19/what-is-the-efficacy-ofstandard-face-masks-compared-to-respirator-masks-in-preventing-covid-typerespiratory-illnesses-in-primary-care-staff/

13. Rollings L. FFP3 respirator face fit testing - what is it all about? $\mathrm{Br}$ Dent J. 2020;229(2): II $2-4$.

14. RPA Bristol, UK. Qualitative Fit Test Kit, Alpha Solway, Bitter. [Internet]. RPA - Face Fit. [cited 2020 Sep I3]. Available from: https://www.face-fit.co.uk/product/rpaqualitative-fit-test-kit/

15. Seligman, Henry, Zaman, Sameer, Pitcher, David, Shun-Shin, Matthew, HepworthLloyd, Freya, Androshuk, Vitaliy, et al. Improved protection against aerosol and droplet deposition using a reusable snorkel mask as particulate respirator. (Under Review).

16. SCBA Sales Co. TSI Portacount Plus Model 8020 w/Accessories (Calibrated) [Internet]. [cited 2020 Sep 13]. Available from: https://www.scbasalesco.com/product/tsiportacount-plus-model-8020-w-accessories-calibrated/

17. Ciotti C, Pellissier G, Rabaud C, Lucet J-C, Abiteboul D, Bouvet E, et al. Effectiveness of respirator masks for healthcare workers, in France. Med Mal Infect. 2012 Jun;42(6):264-9.

18. Hon C-Y, Danyluk Q, Bryce E, Janssen B, Neudorf M, Yassi A, et al. Comparison of qualitative and quantitative fit-testing results for three commonly used respirators in the healthcare sector. J Occup Environ Hyg. 2017;14(3):I75-9.

19. Centers for Disease Control and Prevention. Optimizing Supply of PPE and Other Equipment during Shortages [Internet]. CDC; 2020 [cited 2020 Sep 2]. Available from: https://www.cdc.gov/coronavirus/2019-ncov/hcp/ppe-strategy/index.html

20. Public Health England. Considerations for acute personal protective equipment (PPE) shortages [Internet]. UK Government; 2020 [cited 2020 Sep 2]. Available from: https://www.gov.uk/government/publications/wuhan-novel-coronavirus-infectionprevention-and-control/managing-shortages-in-personal-protective-equipment-ppe

21. Littlewood R, Mollan SP, Pepper IM, Hickman SJ. The Utility of Fundus Fluorescein Angiography in Neuro-Ophthalmology. Neuroophthalmology. 2019 Aug;43(4):2I 7-34. 
22. Calvo CM, Hartnett ME. The utility of ultra-widefield fluorescein angiography in pediatric retinal diseases. Int J Retina Vitreous. 2018;4:2I.

23. Noppen M, Dekeukeleire T, Hanon S, Stratakos G, Amjadi K, Madsen P, et al. Fluorescein-enhanced autofluorescence thoracoscopy in patients with primary spontaneous pneumothorax and normal subjects. Am J Respir Crit Care Med. 2006 Jul I; I 74(1):26-30.

24. Chughtai AA, Stelzer-Braid S, Rawlinson W, Pontivivo G, Wang Q, Pan Y, et al. Contamination by respiratory viruses on outer surface of medical masks used by hospital healthcare workers. BMC Infect Dis. 2019 Jun 3;19(I):49I.

25. Oestenstad RK, Dillion HK, Perkins LL. Distribution of faceseal leak sites on a halfmask respirator and their association with facial dimensions. Am Ind Hyg Assoc J. 1990 May;5 I (5):285-90.

26. Oestenstad RK, Bartolucci AA. Factors affecting the location and shape of face seal leak sites on half-mask respirators. J Occup Environ Hyg. 2010 Jun;7(6):332-4I.

27. Veena HR, Mahantesha S, Joseph PA, Patil SR, Patil SH. Dissemination of aerosol and splatter during ultrasonic scaling: a pilot study. J Infect Public Health. 2015 Jun;8(3):260-5.

28. Health and Safety Executive. Fit Testing of Respiratory Protective Equipment Facepieces [Internet]. HSE; 2012 [cited 2020 Sep 2]. Available from: https://www.nhsggc.org.uk/media/245892/282_28.pdf

29. 3M. Guide to using the $3 M$ Qualitative Fit Test Kits [Internet]. 3M United Kingdom PLC, Bracknell, UK; [cited 2020 Sep 2]. Available from: https://multimedia.3m.com/mws/media/4739600/guide-to-using-the-3m-qualitative-fittest-kits.pdf

30. Schindelin J, Arganda-Carreras I, Frise E, Kaynig V, Longair M, Pietzsch T, et al. Fiji: an open-source platform for biological-image analysis. Nat Methods. 2012 Jun 28;9(7):676-82.

3I. Hammond, Luke. Measuring cell fluorescence using ImageJ [Internet]. The Open Lab Book. 2014 [cited 2020 Jun 12]. Available from:

https://theolb.readthedocs.io/en/latest/imaging/measuring-cell-fluorescence-usingimagej.html

32. Nguyen LH, Drew DA, Graham MS, Joshi AD, Guo C-G, Ma W, et al. Risk of COVID19 among front-line health-care workers and the general community: a prospective cohort study. The Lancet Public Health. 2020 Sep I;5(9):e475-83.

33. Bielicki JA, Duval X, Gobat N, Goossens H, Koopmans M, Tacconelli E, et al. Monitoring approaches for health-care workers during the COVID-I 9 pandemic. The Lancet Infectious Diseases [Internet]. 2020 Jul 23 [cited 2020 Sep 2];0(0). Available from: https://www.thelancet.com/journals/laninf/article/PIIS I473-3099(20)304588/abstract 
34. Centres for Disease Control and Prevention. Criteria for Return to Work for Healthcare Personnel with SARS-CoV-2 Infection (Interim Guidance) [Internet]. CDC; 2020 [cited 2020 Sep 2]. Available from: https://www.cdc.gov/coronavirus/2019ncov/hcp/return-to-work.html

35. Campbell DL, Coffey CC, Jensen PA, Zhuang Z. Reducing respirator fit test errors: a multi-donning approach. J Occup Environ Hyg. 2005 Aug;2(8):39I-9.

\section{Figure and Table Legends (in order of appearance in manuscript)}

Figure I: Study protocol and stages of the fluorescein fit-test. Total fluorescence (TF) measured in $10^{6}$ fluorescence units. Due to each of the taste-test 'fail' and 'pass' groups having even numbers of participants, the TF of the sample filter papers shown in stage ' $E$. Taste-test Results' is not exactly 9.2 and 2.6 respectively. The filter papers shown are those with a TF value closest to the group's medians. The TF of the displayed filter papers are 10.2 and $2.6 \times 10^{6}$ units respectively.

Table I: Baseline characteristics of participants (including stratified by the result of the tastetest). Sensitivity to denatonium benzoate was defined by the number of puffs of $0.02 \%$ denatonium benzoate required for participants to perceive its taste without wearing a mask [ I - I0 puffs = normal sensitivity; I I-20 puffs = low sensitivity; $>20$ puffs = minimal sensitivity].

Figure 2: Box and whisker plots of total fluorescence (TF; measured in $10^{6}$ fluorescence units) of (i) all participants, (ii) participants who 'failed' the taste-test and (iii) participants who 'passed' the taste-test.

Figure 3: Precision and recall of the fluorescein test in comparison to the taste-test for different TF threshold (cut-off) values. The suggested threshold $\left(5.0 \times 10^{6}\right.$ fluorescence units) was selected because at this value both precision (78\%) and recall (84\%) are high, with a preference for maximising recall due to the clinical implications of false positive tests. 


\section{Figures}

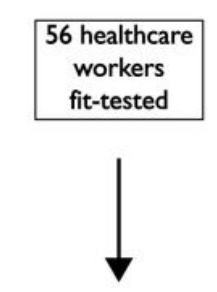

A Test preparation

B Mask fit-test

C Paper preparation

D Image analysis

E Taste-test results

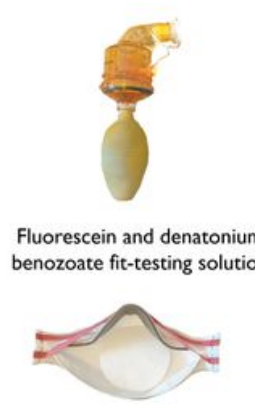

Scientific filter paper placed inside mask

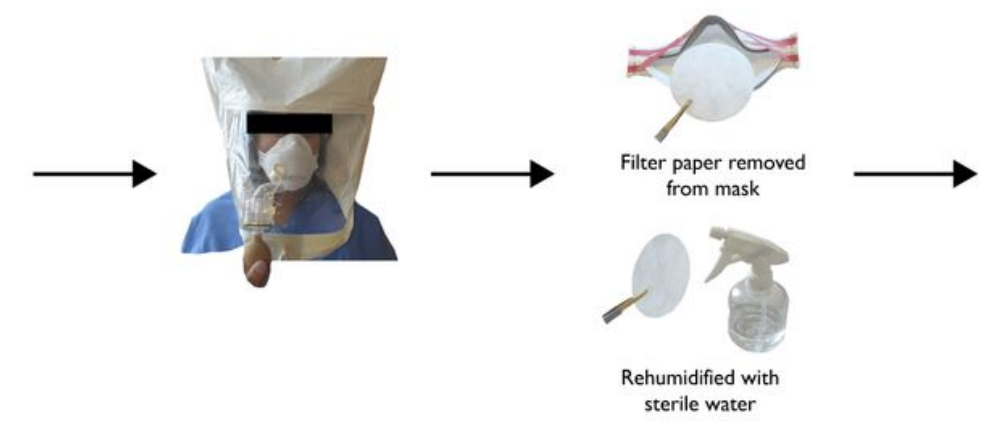

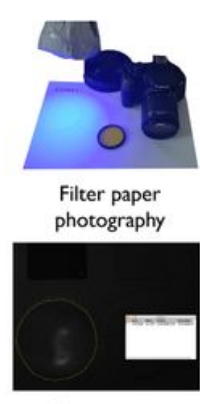

Fluorescence quantification

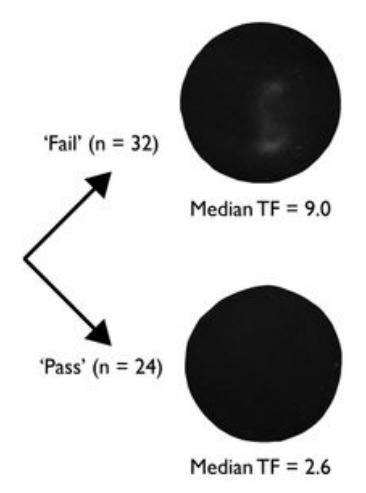

\section{Figure 1}

Study protocol and stages of the fluorescein fit-test. Total fluorescence (TF) measured in $10^{\wedge} 6$ fluorescence units. Due to each of the taste-test 'fail' and 'pass' groups having even numbers of participants, the TF of the sample filter papers shown in stage 'E. Taste-test Results' is not exactly 9.2 and 2.6 respectively. The filter papers shown are those with a TF value closest to the group's medians. The TF of the displayed filter papers are 10.2 and $2.6 \times 10^{\wedge} 6$ units respectively. 


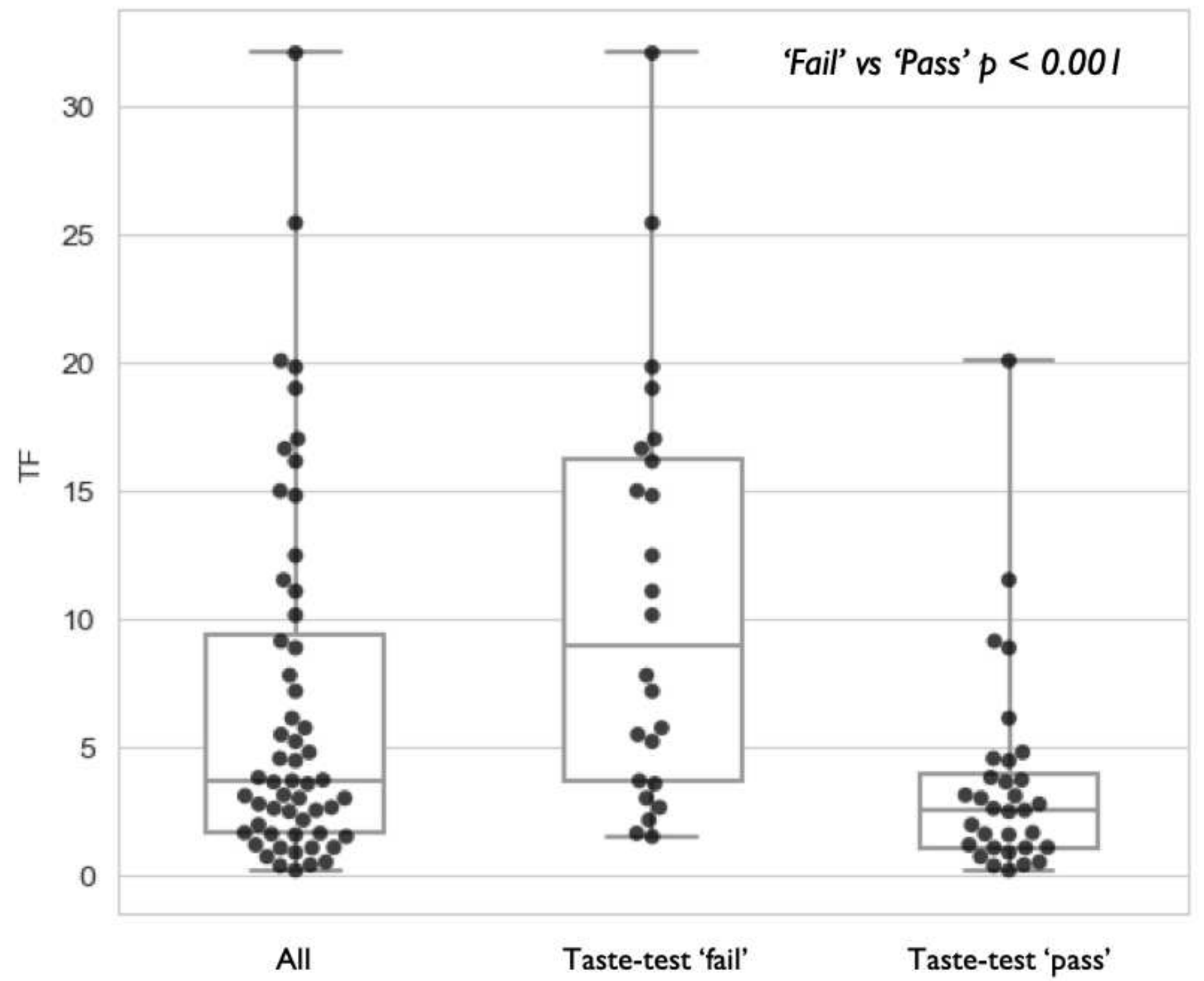

Figure 2

Box and whisker plots of total fluorescence (TF; measured in $10^{\wedge} 6$ fluorescence units) of (i) all participants, (ii) participants who 'failed' the taste-test and (iii) participants who 'passed' the taste-test. 


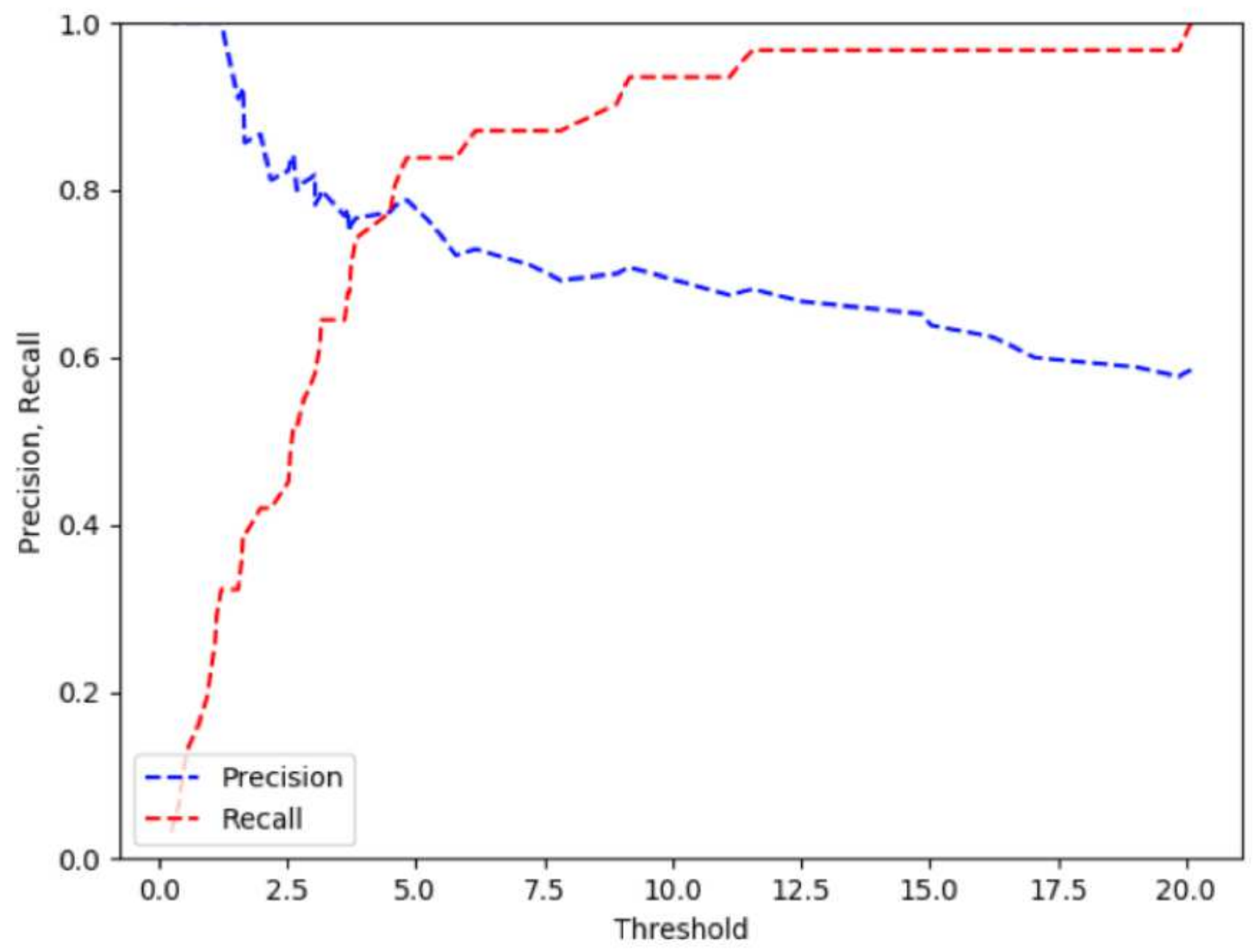

\section{Figure 3}

Precision and recall of the fluorescein test in comparison to the taste-test for different TF threshold (cutoff) values. The suggested threshold $\left(5.0 \times 10^{\wedge} 6\right.$ fluorescence units) was selected because at this value both precision (78\%) and recall (84\%) are high, with a preference for maximising recall due to the clinical implications of false positive tests. 